\title{
Automatic Classification of Normal and Cancer Lung CT Images Using Multiscale AM-FM Features
}

\author{
Eman Magdy, Nourhan Zayed, and Mahmoud Fakhr \\ Computer and Systems Department, Electronic Research Institute, Giza 12611, Egypt \\ Correspondence should be addressed to Eman Magdy; eman.magdy@eri.sci.eg
}

Received 1 May 2015; Revised 24 August 2015; Accepted 1 September 2015

Academic Editor: Tiange Zhuang

Copyright ( 2015 Eman Magdy et al. This is an open access article distributed under the Creative Commons Attribution License, which permits unrestricted use, distribution, and reproduction in any medium, provided the original work is properly cited.

\begin{abstract}
Computer-aided diagnostic (CAD) systems provide fast and reliable diagnosis for medical images. In this paper, CAD system is proposed to analyze and automatically segment the lungs and classify each lung into normal or cancer. Using 70 different patients' lung CT dataset, Wiener filtering on the original CT images is applied firstly as a preprocessing step. Secondly, we combine histogram analysis with thresholding and morphological operations to segment the lung regions and extract each lung separately. Amplitude-Modulation Frequency-Modulation (AM-FM) method thirdly, has been used to extract features for ROIs. Then, the significant AM-FM features have been selected using Partial Least Squares Regression (PLSR) for classification step. Finally, $K$ nearest neighbour $(K N N)$, support vector machine (SVM), naïve Bayes, and linear classifiers have been used with the selected AM-FM features. The performance of each classifier in terms of accuracy, sensitivity, and specificity is evaluated. The results indicate that our proposed CAD system succeeded to differentiate between normal and cancer lungs and achieved 95\% accuracy in case of the linear classifier.
\end{abstract}

\section{Introduction}

Computed Tomography (CT) has outperformed conventional radiography in the screening of lungs because it generates very detailed high-resolution images and can show earlystage lesions that are too small to be detected by conventional $\mathrm{X}$-ray. CT has been widely used to detect numerous lung diseases, including pneumoconiosis, pneumonia, pulmonary edema, and lung cancer [1]. Early detection of diseases is very crucial for treatment planning. However, it is considered one of the most challenging tasks performed by radiologists due to the huge amount of data generated by CT scan. Therefore, computer-aided diagnostic (CAD) systems are needed to assist radiologists in the analysis and evaluation of CT scans.

A CAD system analyzes medical images in several steps: first a preprocessing step for noise reduction and enhancing the image quality and then segmentation step to differentiate region of interest (ROI) from other structures in the image. After segmentation, different features such as geometrical, textural, and statistical features are extracted. Finally, a classification/evaluation step is done to evaluate and diagnose the ROI based on extracted features.

Many efforts have been made to provide computeraided diagnosis for lung images. Lung segmentation is a necessary step; it has progressed from manual tracing to semiautomated to fully automated segmentation. Here, some automated lung segmentation studies are presented [2-9]. Other studies present content-based image retrieval (CBIR) systems for lung images [10-15]. Earlier work in classification of lung cancer includes the work of Patil and Kuchanur [16] and Kuruvilla and Gunavathi [17] that used artificial neural networks to classify lung cancer images based on the features extracted from lung segmented images. Nevertheless, Patil and Kuchanur used geometrical features for classification and achieved only 83\% accuracy of classification. And Kuruvilla and Gunavathi used statistical parameters as features for classification and achieved accuracy of $93.3 \%$. Another work by Depeursinge et al. [18] classified different lung tissue patterns using discrete wavelet frames combined with gray-level histogram features. However, the main limitation of this work was the lack of resolution in scales with the decomposition, 


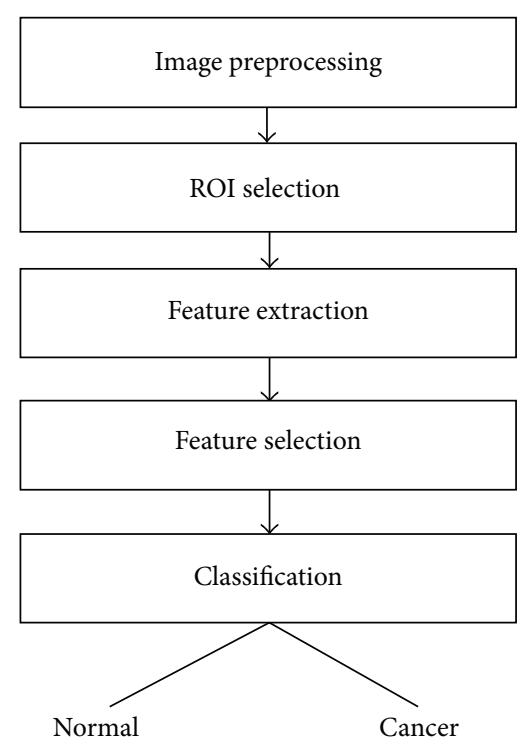

FIGURE 1: Block diagram of the proposed fully automated CAD system.

along with required feature weighting while merging features from different origins.

In this paper, we propose a CAD system for analysis, automatic segmentation, and classification of lung images into normal or cancer from CT dataset. The system is based on the multiscale Amplitude-Modulation FrequencyModulation (AM-FM) approach. The lungs are firstly segmented from CT images and next left and right lungs are separated individually to be analyzed over a filterbank. Then, the AM-FM features are extracted and reduced for the classification step. Different classifiers are used to classify the images and the performance of each classifier has been evaluated.

\section{Materials and Methods}

Figure 1 shows the main block diagram of our proposed fully automated CAD system. As seen in this figure, the system is composed of five main steps: image preprocessing, select region of interest (ROI), feature extraction using AM-FM approach, feature selection to find the significant features, and finally a classification. The details of each step are discussed in the following sections.

2.1. Dataset. Data used in this research were obtained from The Cancer Imaging Archive (TCIA) sponsored by the SPIE, NCI/NIH, AAPM, and the University of Chicago [19]. A dataset of 83 CT images from 70 different patients was included. All images have a size of $512 \times 512$ pixels and are stored in Digital Imaging and Communication in Medicine (DICOM) format. An example of dataset is shown in Figure 2(a). In this figure, the right lung is abnormal as it has a cancer (the rounded gray shape), while the left lung is a normal one. For each lung CT image, we separate the left lung from the right lung automatically (as discussed later in
ROI Selection), and each separated lung is labelled as normal or cancer based on the dataset information.

2.2. Image Preprocessing. The objective of preprocessing step is to remove unwanted noise and enhance image quality. We have used a Wiener filter to remove noise while preserving the edges and fine details of lungs. The filter size of $3 \times 3$ is selected to avoid oversmoothing of the image. The result of Wiener filtering is shown in Figure 2(b).

Wiener filtering [20] is based on estimating the local mean and variance from a local neighborhood of each pixel. Then, it creates pixel-wise linear filtering using these estimates:

$$
F(m, n)=\mu+\frac{\sigma^{2}-v^{2}}{\sigma^{2}}(I(m, n)-\mu),
$$

where $I$ and $F$ denote the original and filtered images, respectively, $\mu$ and $\sigma$ denote the mean and variance of a local neighborhood, respectively, and $v$ is the noise variance.

2.3. ROI Selection. Lung segmentation is a necessary step for any lung CAD system. We perform automatic segmentation of the lungs using successive steps. Then, the resulting segmented image is used to extract each lung separately (ROIs), producing two images: one for the left lung and the other for the right lung.

In the CT image, air appears in a mean intensity of approximately -3024 Hounsfield units (HU), and the lung tissue is in the range of $-910 \mathrm{HU}$ to $-500 \mathrm{HU}$, while other structures are above $-500 \mathrm{HU}$. The goal of segmentation step is to separate the lungs from both background and nonlung regions. To accomplish this, we propose a hybrid technique resulting from a combination of histogram analysis, thresholding, and morphological operations for automatic lung segmentation.

To simplify the segmentation process, the thorax region is firstly segmented from the background. A gray-level distribution (histogram) of the Wiener-filtered image is used to identify different regions in the image. The histogram has one peak corresponding to lung region and another two peaks for fat and muscle of thorax region and lung mediastinum. In addition, there is a spike at $-3024 \mathrm{HU}$ corresponding to background pixels. Figure 3 shows all peaks except for the background spike.

The threshold value is then computed from this histogram according to the following equation:

$$
T=\frac{I_{\mathrm{FM}}-I_{L}}{2}+I_{L}
$$

where $I_{L}$ denotes the peak intensity value of lung region and $I_{\mathrm{FM}}$ denotes the average intensity value of fat/muscle peaks.

Then, a binary image (Figure 4(a)) for segmented thorax region is created where the pixels with gray level greater than the selected threshold are set to "one" and other pixels are "zero."

After the thorax region is segmented, we perform a filling operation to fill the holes inside the binary image, so that the pixel values of lungs change from zero to one and produce 


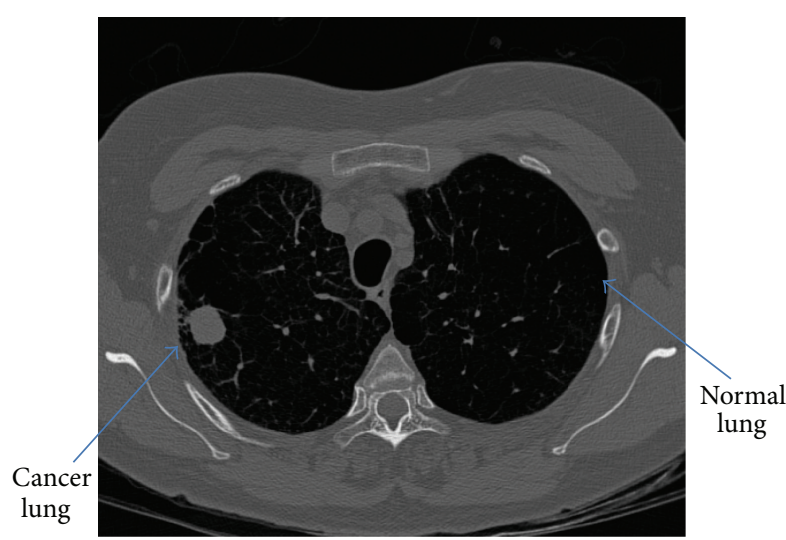

(a)

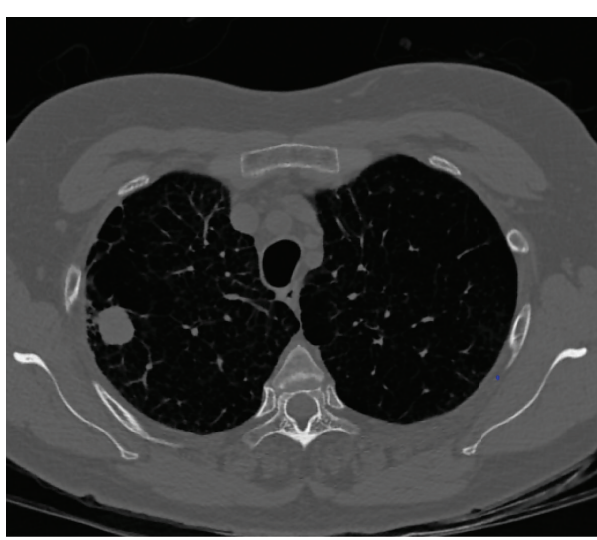

(b)

FIGURE 2: Image preprocessing: (a) original image and (b) filtered image.

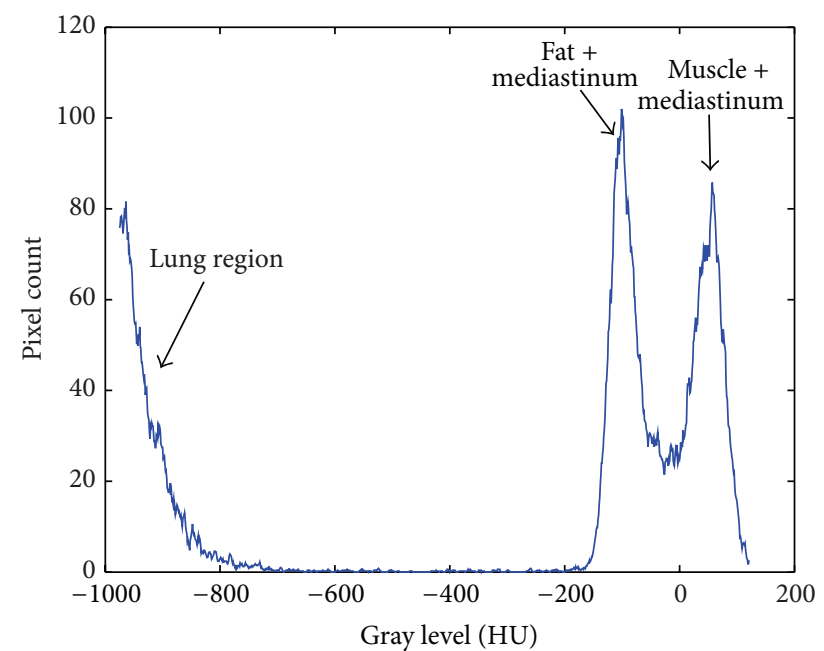

Figure 3: Histogram of the Wiener-filtered image.

a filled image (Figure 4(b)). Finally, the thorax binary image is subtracted from the filled image to obtain the segmented lungs as shown in Figure 4(c).

Once the image of lung segmentation is obtained, we used it to locate the left and right lungs in the filtered image, as seen in Figure 4(d). Then, this image is divided into two images for both lungs separately, each one covering the region of the lung as shown in Figures 4(e) and 4(f). From the $83 \mathrm{CT}$ images, we obtained 166 different lung images after each image division, where 83 of them are normal lung images and the other 83 images are cancer lung images.

2.4. Feature Extraction. In this step, we apply the AmplitudeModulation Frequency-Modulation (AM-FM) modeling techniques to extract features from lung images that will be used further in classification. A lot of research work has been made on AM-FM models [21-23].

2.4.1. AM-FM Methods. AM-FM is a technique that models nonstationary signals. Unlike Fourier transforms that provide the frequency content of signal, AM-FM methods provide pixel-based information in terms of instantaneous amplitude (IA), instantaneous frequency (IF), and instantaneous phase (IP). And it does not have the main limitation found on wavelet when used to segment the lungs which was the lack of resolution in scales with the decomposition.

In 2D AM-FM model, a nonstationary image is represented by a sum of AM-FM components as [22]

$$
I\left(k_{1}, k_{2}\right)=\sum_{n=1}^{M} a_{n}\left(k_{1}, k_{2}\right) \cos \varphi_{n}\left(k_{1}, k_{2}\right),
$$

where $n=1,2, \ldots, M$ denotes the different AM-FM harmonics, $a_{n}\left(k_{1}, k_{2}\right)$ denotes the instantaneous amplitude functions (IA), and $\varphi_{n}\left(k_{1}, k_{2}\right)$ denotes the instantaneous phase functions (IP).

For each AM-FM component, the instantaneous frequency (IF) is defined as the gradient of phase $\nabla \varphi_{n}\left(k_{1}\right.$, $\left.k_{2}\right)=\left(\partial \varphi_{n}\left(k_{1}, k_{2}\right) / \partial k_{1}, \partial \varphi_{n}\left(k_{1}, k_{2}\right) / \partial k_{2}\right)$. Here, the AM-FM demodulation problem is to estimate the IA, IF, and IP for the given input image.

In this work, AM-FM demodulation is achieved in several steps. First, we extend the input image to an analytic image by adding an imaginary part equal to the 2D Hilbert transform of the image [21]. Given a real-valued image $I\left(k_{1}, k_{2}\right)$, the analytic image $I_{\mathrm{AS}}\left(k_{1}, k_{2}\right)$ is calculated as follows:

$$
I_{\mathrm{AS}}\left(k_{1}, k_{2}\right)=I\left(k_{1}, k_{2}\right)+j H_{2 \mathrm{D}}\left[I\left(k_{1}, k_{2}\right)\right] \text {, }
$$

where $H_{2 \mathrm{D}}$ denotes a two-dimensional extension of onedimensional Hilbert transform.

Then, the analytic image is processed through a collection of band-pass filters (filterbank) (to be discussed in the next subsection) in order to isolate the AM-FM components.

And, from each filter response, we can estimate the IA and IP straightforwardly using these following equations:

$$
\begin{aligned}
& \widehat{a}\left(k_{1}, k_{2}\right)=\left|I_{\mathrm{AS}}\left(k_{1}, k_{2}\right)\right|, \\
& \widehat{\varphi}\left(k_{1}, k_{2}\right)=\tan ^{-1} \frac{\operatorname{imag}\left(I_{\mathrm{AS}}\left(k_{1}, k_{2}\right)\right)}{\operatorname{real}\left(I_{\mathrm{AS}}\left(k_{1}, k_{2}\right)\right)} .
\end{aligned}
$$




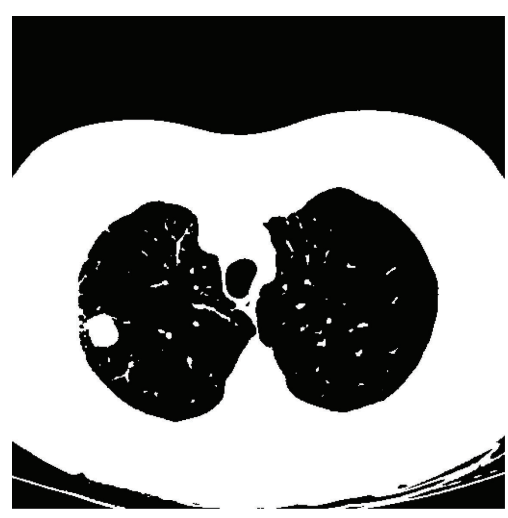

(a)

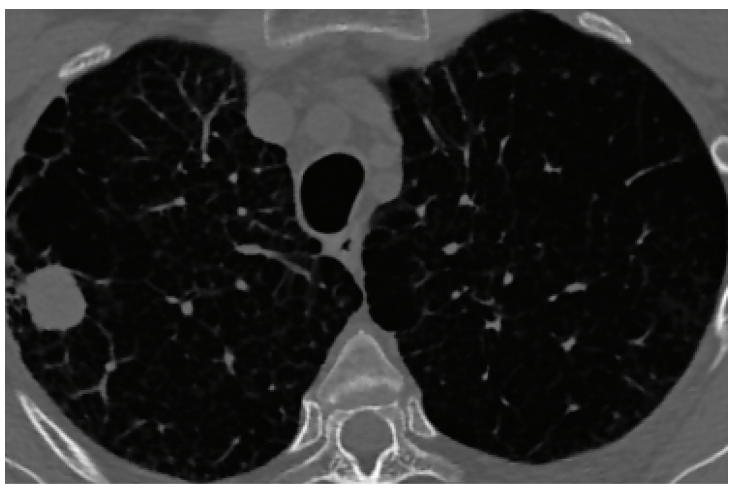

(d)
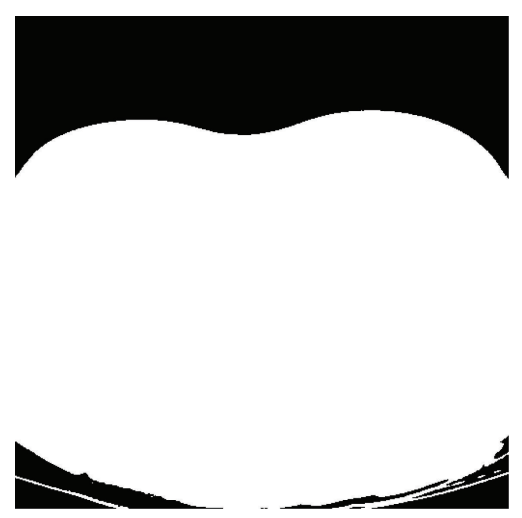

(b)

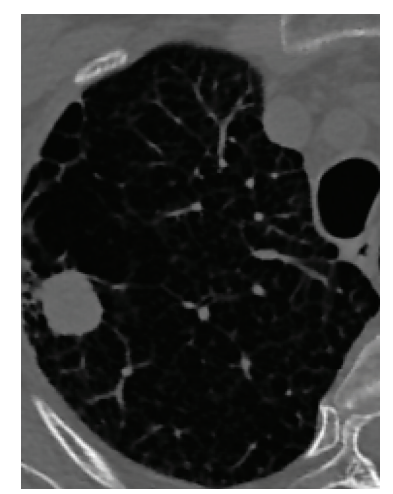

(e)

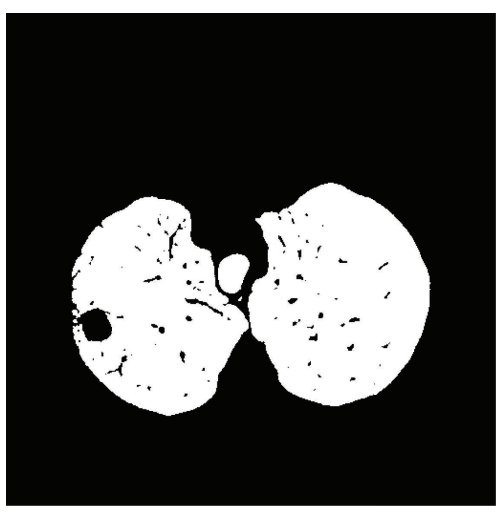

(c)

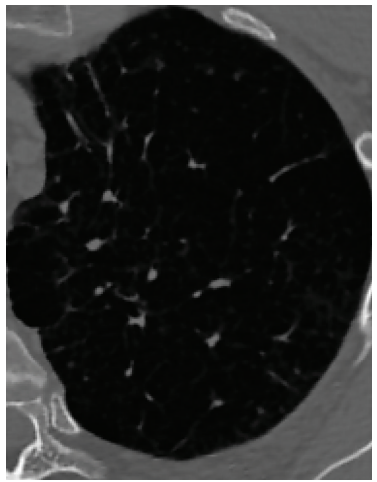

(f)

FIGURE 4: Segmentation and ROI selection. (a) Thorax binary image. (b) Filled image. (c) Lung segmentation. (d) Rectangular ROI of both lungs. (e) Right lung (cancer). (f) Left lung (normal).

To estimate the IF, we used a variable spacing local linear phase (VS-LLP) method as described in [23].

2.4.2. Filterbank Design. The purpose of multiscale filterbank is to isolate the AM-FM image components in model (3) prior to performing demodulation. Here, we use a four-scale filterbank developed by Murray [22] (see Figure 5).

In Figure 5, the frequency range of filterbank is depicted. Filter 1 is a low-pass filter (LPF), filters 2-7 are high frequency filters $(H)$, filters $8-13$ are medium frequency filters $(M)$, filters 14-19 are low frequency filters (L), and filters 20-25 are very low frequency filters (VL). It can be noticed that the bandwidth is decreased by a factor of $1 / 2$ for each added scale.

In this paper, we used different combinations of scales to extract the dominant AM-FM features. Here are the combinations used: (1) VL, L, M, and H; (2) LPF; (3) VL; (4) L; (5) M; (6) LPF, VL, L, M, and H; (7) LPF, VL; (8) VL, L; (9) L, M; (10) M, H; and (11) H. And, for each combination of scales, we estimate the IA, IP, and |IF| using (5) and the equations in [23].

2.4.3. Histogram Processing. For each combination of scales, we produce a histogram for AM-FM estimates: IA, IP, and $|\mathrm{IF}|$. And all the computed histograms are normalized so that the area of each histogram is equal to one. Then, for each combination of scales, we create a 96-bin feature vector from the IA, IP, and $|\mathrm{IF}|$ histograms, with 32 bins for IA, 32 bins for IF magnitude, and 32 bins for IP (centered at the maximum value). Therefore, each image produces 11 feature vectors corresponding to the 11 combinations of scales. We need to obtain a combined feature vector for each case by selecting the optimal and signification features from all over scales. Thus, we use Partial Least Squares Regression (PLSR) to achieve that.

2.4.4. Feature Selection. Feature selection is an important step that provides the significant features, which are used to differentiate between different classes accurately. We used Partial Least Squares Regression (PLSR) [24], which is a linear regression method that finds the relation between the predicted variables and observations. The regression problem is defined as

$$
y=X \beta+\varepsilon,
$$

where $X$ is an $n \times p$ matrix of the extracted AM-FM features ( $n$ is the number of images and $p$ is the number of features) and $y$ is an $n \times 1$ vector of response or labels. We used label 0 for normal case and label 1 for abnormal case. $\beta$ is $p \times 1$ vector of the regression parameters and $\varepsilon$ is $n \times 1$ vector of the residuals.

We apply PLSR to determine the optimal number of features to be used. We select the PLSR factors number that 


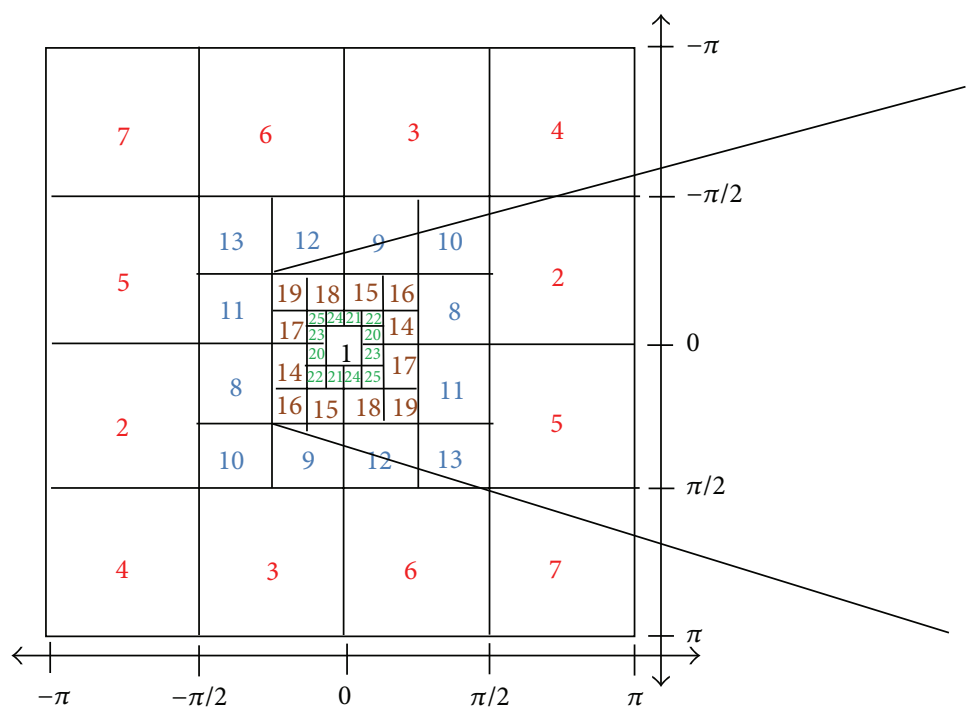

(a)

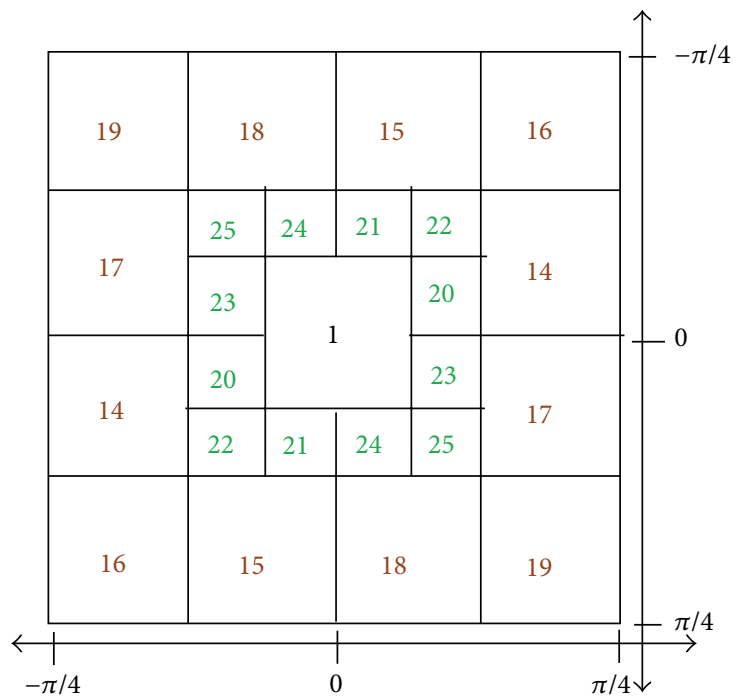

(b)

Figure 5: Four-scale filterbank [22]. (a) Complete frequency range of the filterbank. (b) Zoom on the low frequency filters (to be easily readable).

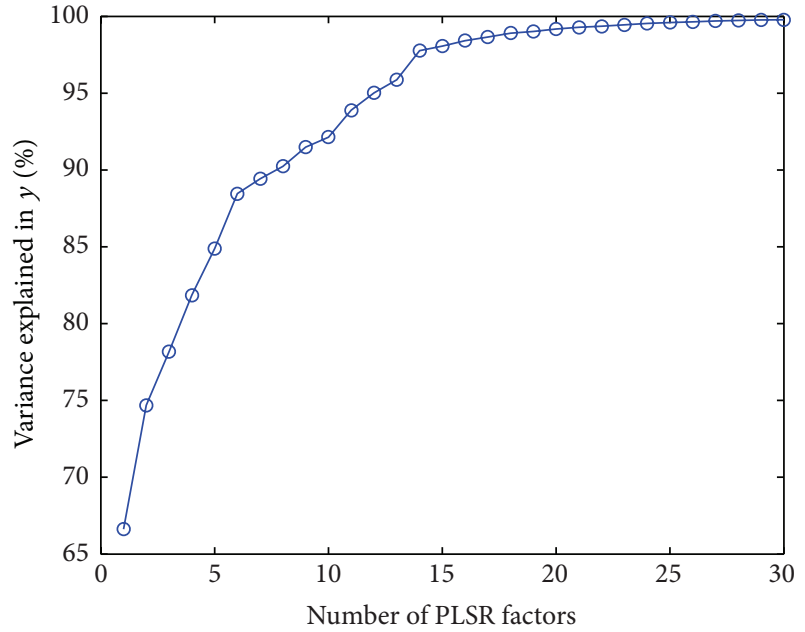

Figure 6: Plotting the number of PLSR factors with the cumulative variance percentage in the response variable $y$.

produces percentage of variance in response variable more than $90 \%$. In Figure 6, we plot the percentage of variance in the response versus the number of PLSR factors. The plot shows that nearly $90 \%$ of the variance in $y$ is given by the first eleven factors.

Once the optimal number of features is obtained, we form a feature vector to represent the selected features.

2.5. Classification. The final step of the proposed system is to correctly discriminate between normal and cancer lung images. The input to classification stage is the feature matrix from the previous step and the labeled vector (where $0=$ normal and $1=$ cancer).
Here, we have used four different classifiers: $K$-nearest neighbor $(K N N)$ [25], support vector machine (SVM) [26], naive Bayes [25], and linear classifier [25]. The basic idea of all of these classifiers depends on supervised learning; that is, each classifier takes a set of labeled images as a training set to build a model that is used further to assign new images (testing set) into classes. Out of 166 lung images, 100 images are selected as a training dataset and 66 other images are selected as a testing dataset.

\section{Results and Discussion}

The four classifiers are trained and their performance is evaluated with leave- $M$-out cross validation. We change the value of $M$ to generate different sizes of testing and training sets, and, for each $M$ value, the classification performance is evaluated by computing these different measures:

$$
\begin{aligned}
& \text { Sensitivity }(\%)=\frac{\mathrm{TP}}{\mathrm{TP}+\mathrm{FN}} \times 100, \\
& \text { Specificity }(\%)=\frac{\mathrm{TN}}{\mathrm{FP}+\mathrm{TN}} \times 100, \\
& \operatorname{Accuracy}(\%)=\frac{\mathrm{TP}+\mathrm{TN}}{\mathrm{TP}+\mathrm{FN}+\mathrm{TN}+\mathrm{FP}} \times 100,
\end{aligned}
$$

where TP, TN, FN, and FP denote true positive, true negative, false negative, and false positive, respectively [27].

Figure 7 shows the computed accuracy, sensitivity, and specificity, respectively, for the four classifiers with the change in size of testing set. It can be noticed that the classifiers performances in terms of accuracy, sensitivity, and specificity are much better in case of small size of the testing set (when the classifiers got trained with large size of the training set). However, the performances of all classifiers decrease 

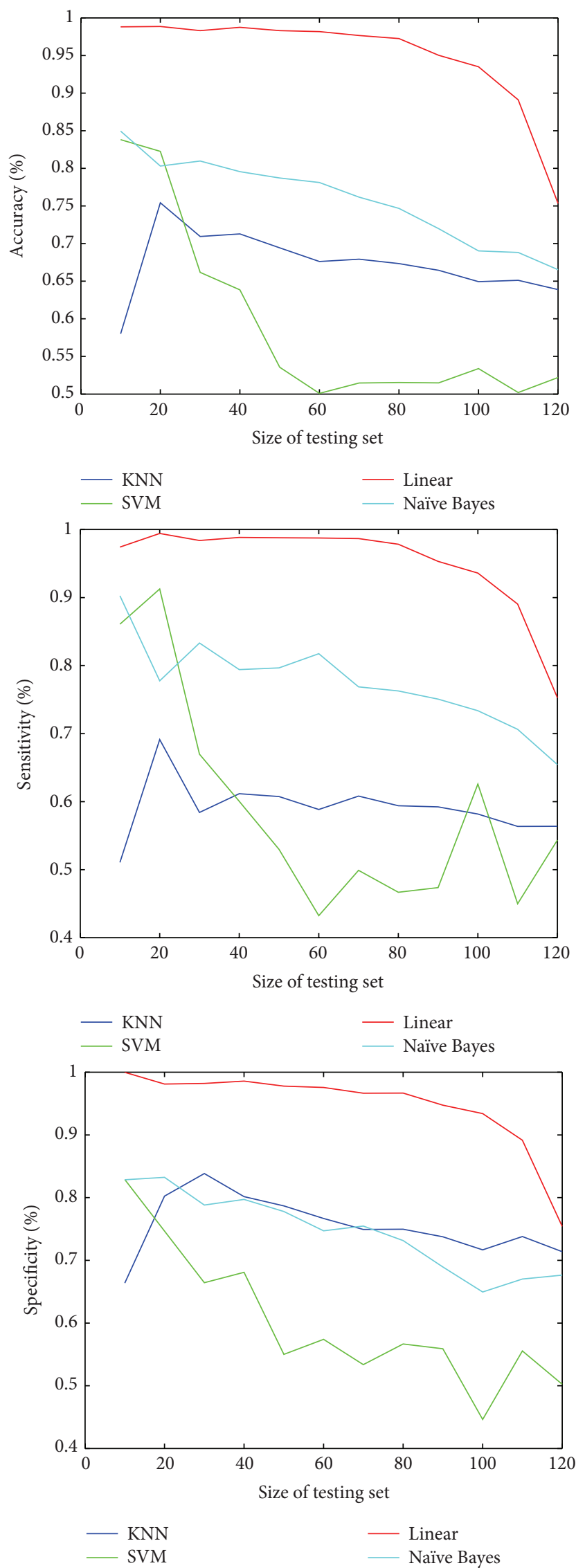

FIGURE 7: Comparison between accuracy, sensitivity, and specificity for the four classifiers with changing size of testing set.
TABLE 1: Classification performance measures for the four classifiers.

\begin{tabular}{lccc}
\hline Classifier & Accuracy & Sensitivity & Specificity \\
\hline KNN & $64 \%$ & $55 \%$ & $72 \%$ \\
SVM & $90 \%$ & $85 \%$ & $97 \%$ \\
Naïve Bayes & $82 \%$ & $82 \%$ & $82 \%$ \\
Linear & $95 \%$ & $94 \%$ & $97 \%$ \\
\hline
\end{tabular}

with increasing testing set size. From this figure, it is easily observed that the performance of SVM classifier is the least stable with increasing testing set size, while the other three classifiers are more stable. Moreover, it can be concluded that the linear classifier is the best one to discriminate between normal and cancer lungs.

Table 1 summarizes the performance measures for the four classifiers when the size of training set relative to the testing set is $60 \%$ to $40 \%$ of the total dataset size, respectively (i.e., training set $=100$ images and test set $=66$ images). As shown in Table 1, the linear classifier gives the best classification with 95\% accuracy, 94\% sensitivity, and 97\% specificity. On the other hand, the KNN classifier is the worst classifier achieving only $64 \%$ accuracy, $55 \%$ sensitivity, and $72 \%$ specificity.

It is worth noting that the proposed CAD system is developed using MATLAB R2010a on Intel Core i5, $2.5 \mathrm{GHz}$, CPU, 6 GB RAM, Windows 7 64-bit PC.

\section{Conclusions}

In this paper, we develop a lung CAD system that analyzes and automatically segments the lungs and classifies each lung to normal or cancer. The system consists of five main steps: preprocessing, ROI selection, feature extraction, feature selection, and classification. AM-FM approach has been used to extract new features in terms of IA, IP, and IF. And PLSR is then used to reduce the large number of features and select the optimal and significant ones. Four classifiers are used and the performance of each classifier has been evaluated. It has been found that the linear classifier was the best one to discriminate between normal and cancer lungs with $95 \%$ accuracy.

\section{Conflict of Interests}

The authors declare that there is no conflict of interests regarding the publication of this paper.

\section{Acknowledgments}

The authors acknowledge the SPIE, the NCI, the AAPM, and The University of Chicago for making public access to the lung cancer dataset.

\section{References}

[1] N. Hollings and P. Shaw, "Diagnostic imaging of lung cancer," European Respiratory Journal, vol. 19, no. 4, pp. 722-742, 2002. 
[2] W. Li, S. D. Nie, and J. J. Cheng, "A fast automatic method of lung segmentation in CT images using mathematical morphology," in World Congress on Medical Physics and Biomedical Engineering 2006, vol. 14 of IFMBE Proceedings, pp. 2419-2422, Springer, Berlin, Germany, 2007.

[3] S. Hu, E. A. Hoffman, and J. M. Reinhardt, "Automatic lung segmentation for accurate quantitation of volumetric X-ray CT images," IEEE Transactions on Medical Imaging, vol. 20, no. 6, pp. 490-498, 2001.

[4] J. K. Leader, B. Zheng, R. M. Rogers et al., "Automated lung segmentation in X-ray computed tomography: development and evaluation of a heuristic threshold-based scheme," Academic Radiology, vol. 10, no. 11, pp. 1224-1236, 2003.

[5] M. N. Prasad, M. S. Brown, S. Ahmad et al., "Automatic segmentation of lung parenchyma in the presence of diseases based on curvature of ribs," Academic Radiology, vol. 15, no. 9, pp. 1173-1180, 2008.

[6] J. Wang, F. Li, and Q. Li, "Automated segmentation of lungs with severe interstitial lung disease in CT," Medical Physics, vol. 36, no. 10, pp. 4592-4599, 2009.

[7] S. Armato and H. MacMahon, "Automated lung segmentation and computer-aided diagnosis for thoracic CT scans," International Congress Series, vol. 1256, pp. 977-982, 2003.

[8] S. G. Armato III and W. F. Sensakovic, "Automated lung segmentation for thoracic CT: impact on computer-aided diagnosis," Academic Radiology, vol. 11, no. 9, pp. 1011-1021, 2004.

[9] Y. Guo, Y. Feng, J. Sun et al., "Automatic lung tumor segmentation on PET/CT images using fuzzy markov random field model," Computational and Mathematical Methods in Medicine, vol. 2014, Article ID 401201, 6 pages, 2014.

[10] M. Lam, T. Disney, M. Pham, D. Raicu, J. Furst, and R. Susomboon, "Content-based image retrieval for pulmonary computed tomography nodule images," in Medical Imaging 2007: PACS and Imaging Informatics, vol. 6516 of Proceedings of SPIE, International Society for Optics and Photonics, March 2007.

[11] A. K. Dhara, C. K. Chama, S. Mukhopadhyay, and N. Khandelwal, "Content-based image retrieval system for differential diagnosis of lung cancer," Indian Journal of Medical Informatics, vol. 6, no. 1, article 1, 2012.

[12] J. B. Nirmala and S. Gowri, "A content based CT lung image retrieval by DCT matrix and feature vector technique," International Journal of Computer Science Issues, vol. 9, no. 2, 2012.

[13] H. Müller, N. Michoux, D. Bandon, and A. Geissbuhler, "A review of content-based image retrieval systems in medical applications-clinical benefits and future directions," International Journal of Medical Informatics, vol. 73, no. 1, pp. 1-23, 2004.

[14] C.-T. Liu, P.-L. Tai, A. Y.-J. Chen, C.-H. Peng, T. Lee, and J.S. Wang, "A content-based CT lung image retrieval system for assisting differential diagnosis images collection," in Proceedings of the IEEE International Conference on Multimedia and Expo (ICME 2001), pp. 174-177, August 2001.

[15] Y. Song, W. Cai, S. Eberl, M. J. Fulham, and D. Feng, "A content-based image retrieval framework for multi-modality lung images," in Proceedings of the 23rd IEEE International Symposium on Computer-Based Medical Systems (CBMS '10), pp. 285-290, October 2010.

[16] S. A. Patil and M. B. Kuchanur, "Lung cancer classification using image processing," International Journal of Engineering and Innovative Technology, vol. 2, no. 3, 2012.
[17] J. Kuruvilla and K. Gunavathi, "Lung cancer classification using neural networks for CT images," Computer Methods and Programs in Biomedicine, vol. 113, no. 1, pp. 202-209, 2014.

[18] A. Depeursinge, D. Sage, A. Hidki et al., "Lung tissue classification using wavelet frames," in Proceedings of the 29th Annual International Conference of IEEE Engineering in Medicine and Biology Society (EMBC '07), pp. 6259-6262, IEEE, Lyon, France, August 2007.

[19] SPIE-AAPM-NCI Lung Nodule Classification Challenge Dataset, The Cancer Imaging Archive, 2015.

[20] H. Shao, L. Cao, and Y. Liu, "A detection approach for solitary pulmonary nodules based on CT images," in Proceedings of the 2nd International Conference on Computer Science and Network Technology (ICCSNT '12), pp. 1253-1257, Changchun, China, December 2012.

[21] J. Havlicek, AM-FM image models [Ph.D. thesis], University of Texas at Austin, Austin, Tex, USA, 1996.

[22] V. Murray, AM-FM methods for image and video processing [Ph.D. thesis], University of New Mexico, Albuquerque, NM, USA, 2008.

[23] V. Murray, P. Rodriguez, and M. S. Pattichis, "Multiscale AMFM demodulation and image reconstruction methods with improved accuracy," IEEE Transactions on Image Processing, vol. 19, no. 5, pp. 1138-1152, 2010.

[24] R. Rosipal and N. Krämer, "Overview and recent advances in partial least squares," in Subspace, Latent Structure and Feature Selection, vol. 3940, pp. 34-51, Springer, 2006.

[25] T. Mitchell, Machine Learning, 1997.

[26] C. Campbell and Y. Ying, Learning with Support Vector Machines, 2011.

[27] N. A. Papadopoulos, E. M. Plissiti, and I. D. Fotiadis, "Medicalimage processing and analysis for CAD systems," in Medical Image Analysis Methods, L. Costaridou, Ed., pp. 51-86, Taylor \& Francis, CRC Press, Boca Raton, Fla, USA, 2005. 

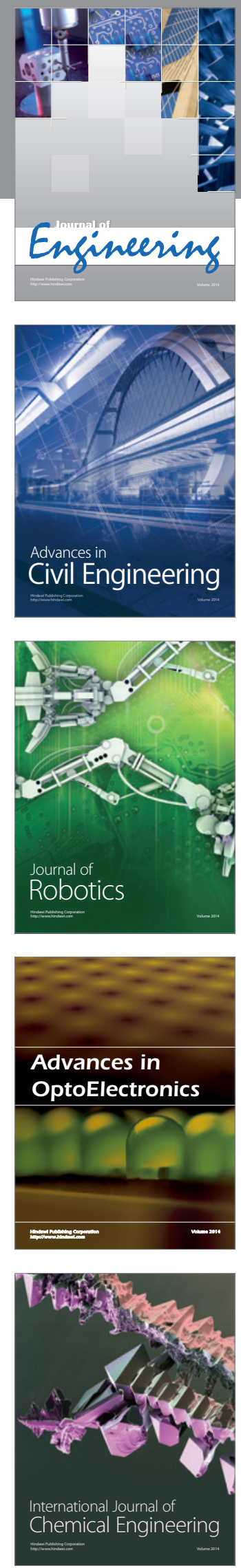

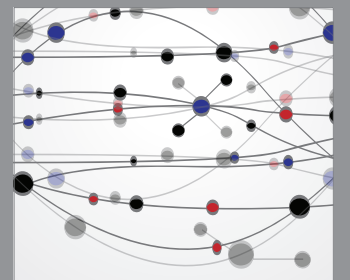

The Scientific World Journal
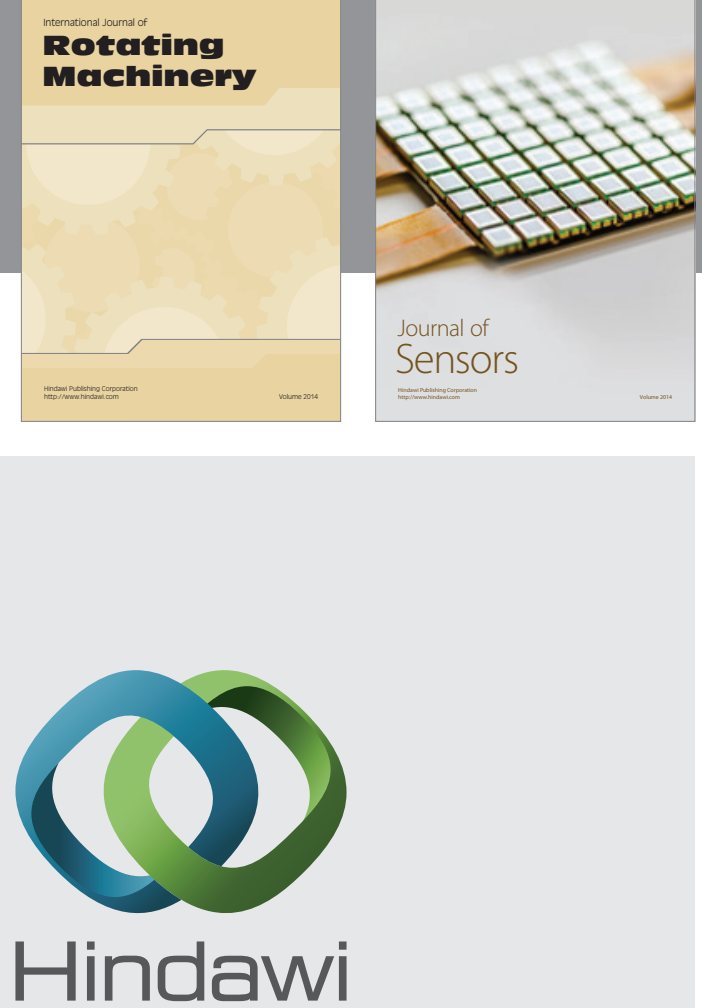

Submit your manuscripts at http://www.hindawi.com
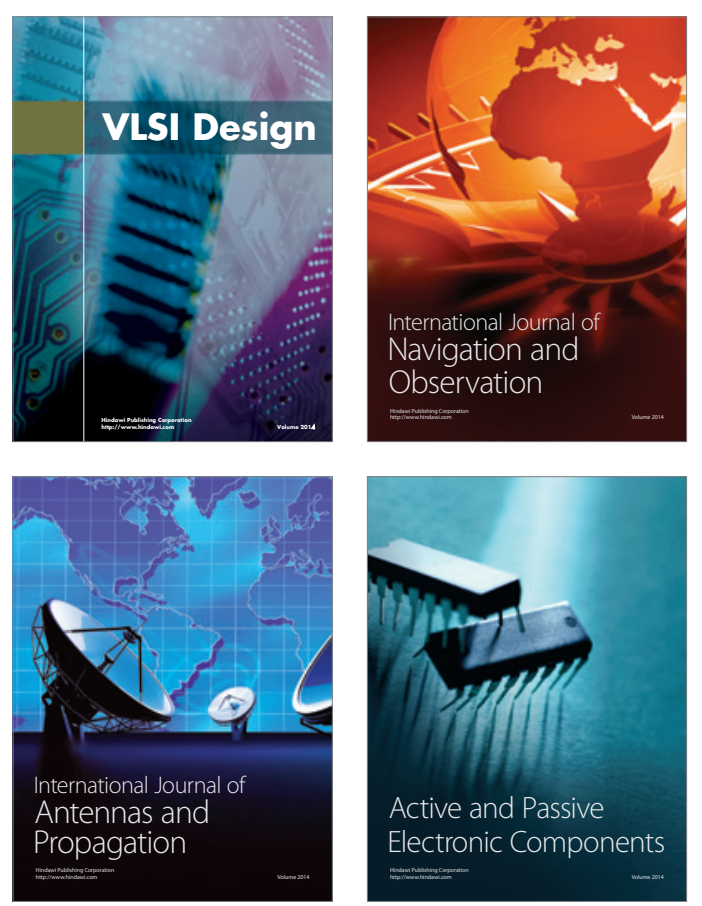
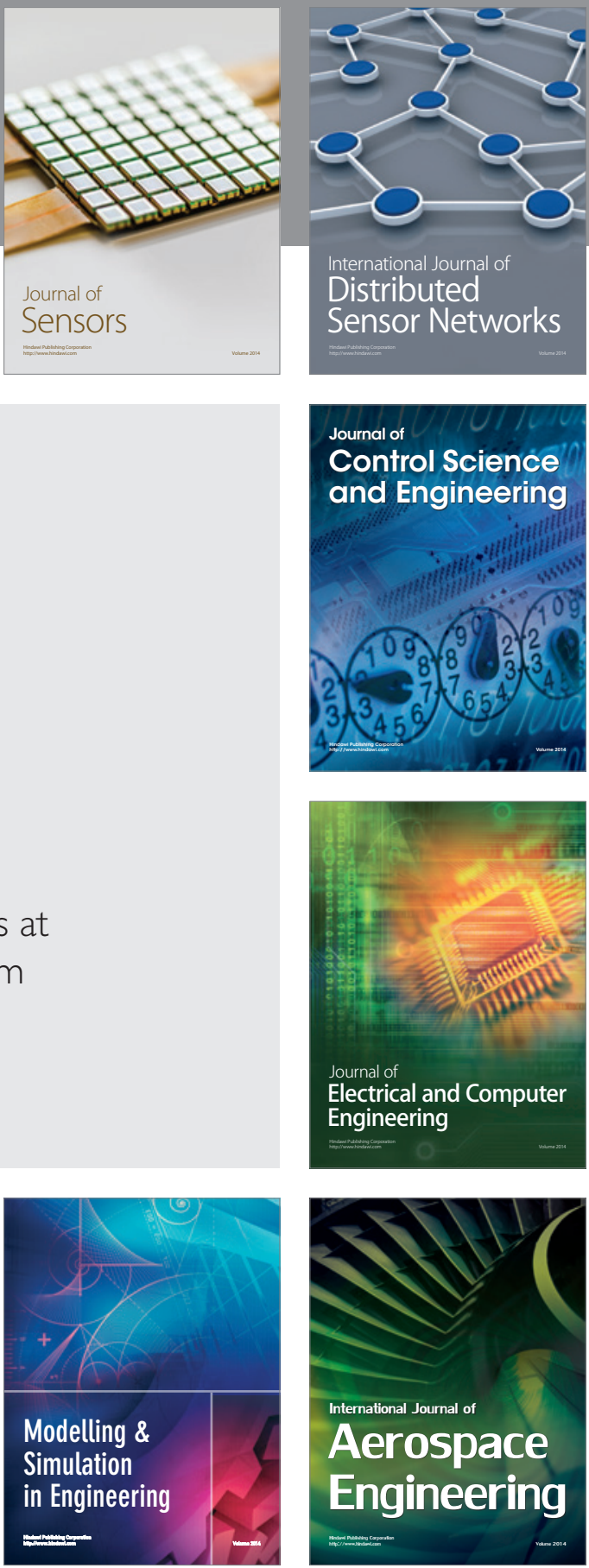

Journal of

Control Science

and Engineering
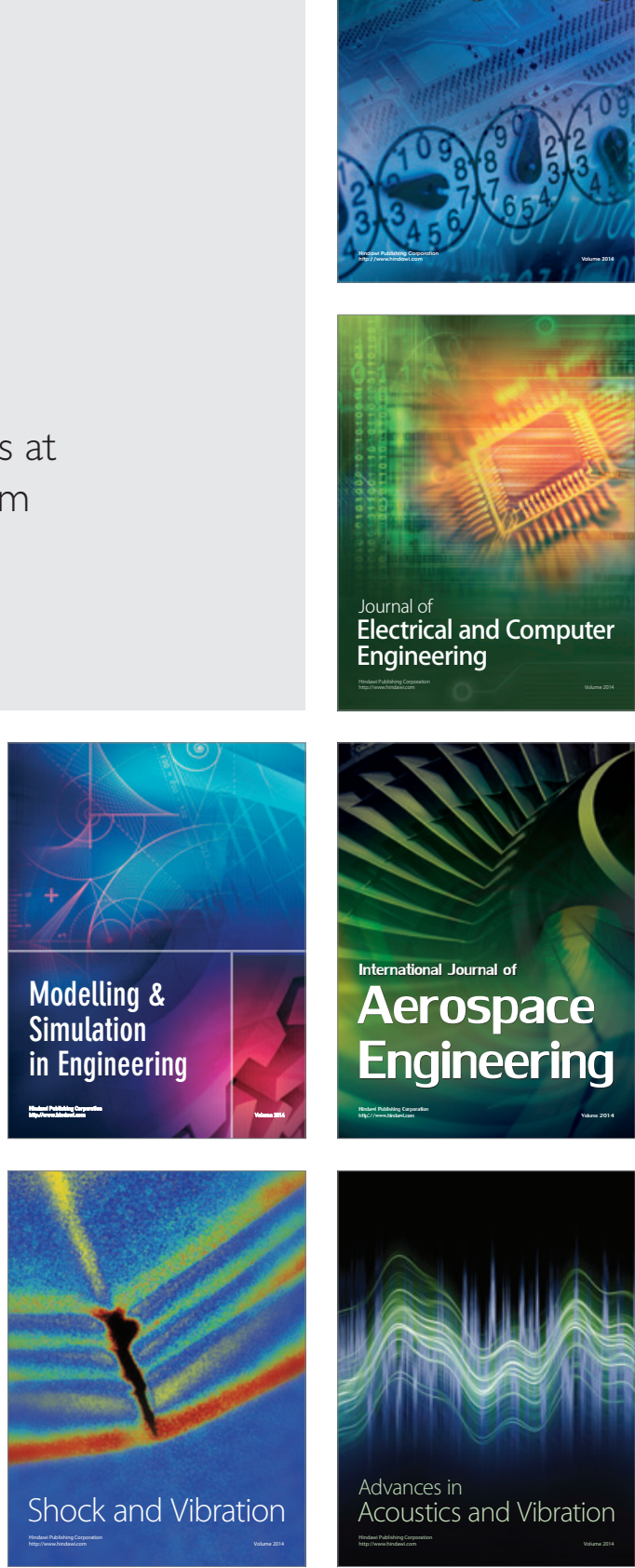